\title{
Venous Occlusion Detected by Caregiver with Implantable Doppler in a Buried Free Flap
}

\author{
Su Won Hur, \\ Tae Gon Kim, \\ Jun Ho Lee, \\ Kyu Jin Chung, \\ Yong-Ha Kim \\ Department of Plastic and Reconstructive \\ Surgery, Yeungnam University College of \\ Medicine, Daegu, Korea \\ No potential conflict of interest relevant to \\ this article was reported.
}

\begin{abstract}
The use of the implantable Doppler device eases the burden of free flap monitoring, and allows caregivers to notify healthcare personnel of a potential vascular event. A 24-year-old female patient underwent anterolateral thigh adipofascial flap surgery to provide a buried flap on the left temporal area for a depressed and infected skull wound. The author was able to salvage the flap from two venous occlusions, which was made possible by early notifications from the caregiver who reported changes in the Doppler signal.
\end{abstract}

Keywords: Free tissue flaps/Caregivers/Pulsed Doppler ultrasonography

\section{INTRODUCTION}

In salvaging compromised free flaps, expedited exploration of vascular anastomosis is only possible with timely discovery of the vascular occlusion. As such, frequent clinical surveillance by medical personnel is mandatory but free flaps without any visible skin island cannot be evaluated through simple physical methods such as skin color, capillary refill, skin turgor, temperature, and prickling [1]. Furthermore, real-time flap monitoring by medical personnel is cost prohibitive from an economic standpoint. The use of implantable Doppler device allows for monitoring of buried free flaps through audible and visible feedback [2]. Flap-compromising vascular events can be appreciated by non-healthcare professionals who are present such as family members or friends of patients. Here, we report successful salvage of a compromised

\section{Correspondence: Tae Gon Kim}

Department of Plastic and Reconstructive Surgery, Yeungnam University College of Medicine, 170 Hyeonchung-ro, Nam-gu, Daegu 705-717, Korea E-mail: kimtg0919@hanmail.net

*This article was supported by Yeungnam University grants in 2013.

*This article was presented as free paper at the 71th Congress of the Korean Society of Plastic and Reconstructive Surgeons on November 1-3, 2013 in Seoul, Korea.

Received June 30, 2014 / Revised July 30, 2014 / Accepted October 15, 2014 buried free flap with a long vascular pedicle. In two sequential venous occlusion events, the hospital staff was notified by a caregiver who perceived the Doppler signal to have changed at each event.

\section{CASE REPORT}

A 24-year-old female patient underwent a buried anterolateral thigh adipofascial free flap transfer to a depressed and infected wound in the left temporal area (Fig. 1). Previously, she had received a cranioplasty with artificial bone for a subdural abscess. Because the superficial temporal vessels had been compromised by previous operations, facial vessels needed to be used as the recipient vessels. The vascular pedicle to the buried flap was lengthened with the contralateral descending branch of the lateral femoral circumflex vessel (Fig. 2). An implantable Doppler system (CookSwartz Doppler) was implemented because the buried flap was at high risk of venous occlusion with such a long vascular pedicle. The ultrasound probe was attached loosely wrapped around the distal portion of venous anastomosis (Fig. 3). Postoperatively, the flap was checked every two hours by the resident staff. In the intervening hours, patient caregivers were simply asked to provide notification of a change in, or the loss of Doppler signal (Fig. 4). The sig- 

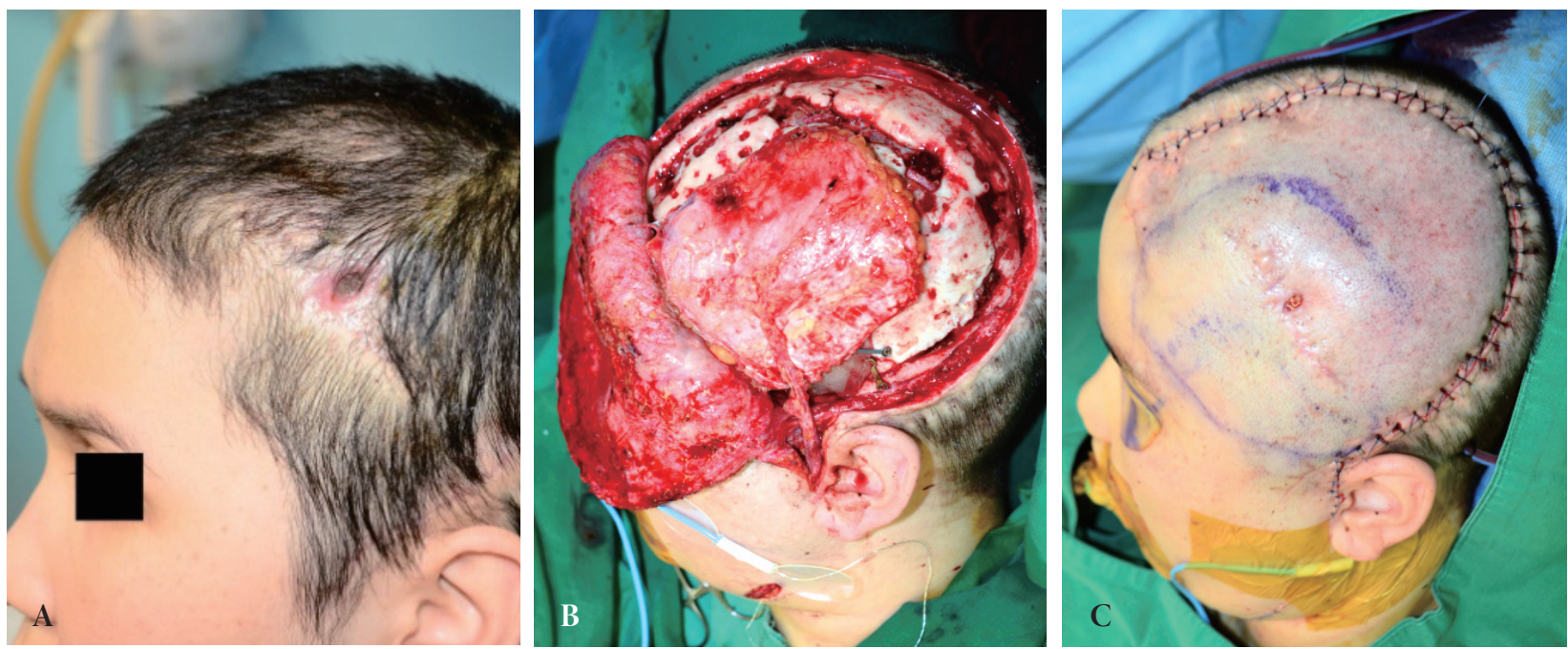

Fig. 1. A 24-year-old female patient was referred from a neurosurgeon for a depressed and infected skull wound on the left temporal area. Previously, the patient had two cranioplasty operations for a subdural abscess. (A) Preoperative view. (B) Intraoperative view. (C) Postoperative view.

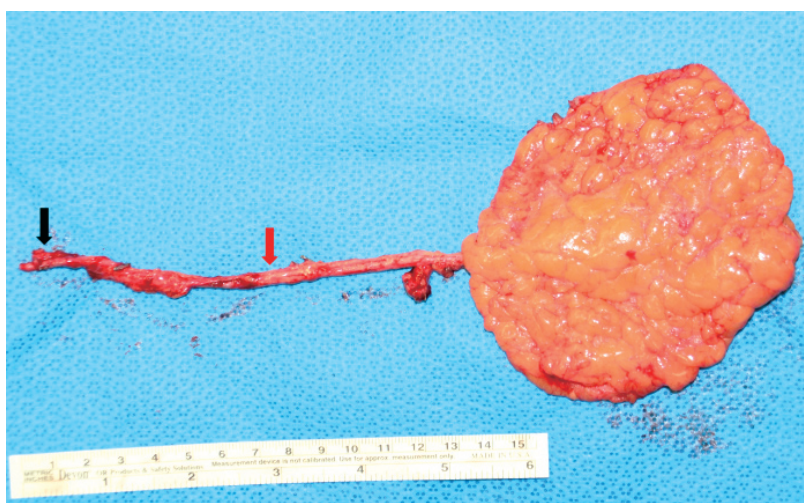

Fig. 2. An anterolateral thigh adipofascial flap was harvested from the left thigh. The descending branch of the left lateral femoral circumflex vessel measured $10 \mathrm{~cm}$ (red arrow). The descending branch of the right circumflex vessel (black arrow) was used to lengthen the pedicle (black arrow). The combined pedicle length was $17 \mathrm{~cm}$.

nal of implantable Doppler suddenly changed over a 15 minuteperiod, which the patient's mother first noticed and reported to the hospital staff. The patient underwent two sequential salvage operations (Fig. 5). The first revision was performed 18 hours after the original reconstruction surgery; venous anastomosis was performed using the lesser saphenous vein graft. The second operation was performed 62 hours after the first revision to remove a thrombus. The implantable Doppler was used in both revisional surgeries, and both events were initially observed by a caregiver. After then, the condition of the flap was recovered and the patient could be discharged (Fig. 6).

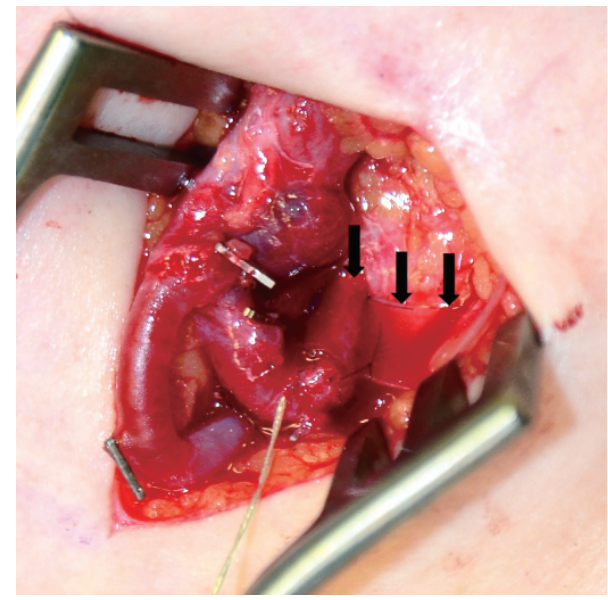

Fig. 3. The implantable Doppler probe was attached to a silastic cuff (black arrows), which was wrapped loosely around the vein distal to the anastomotic point.

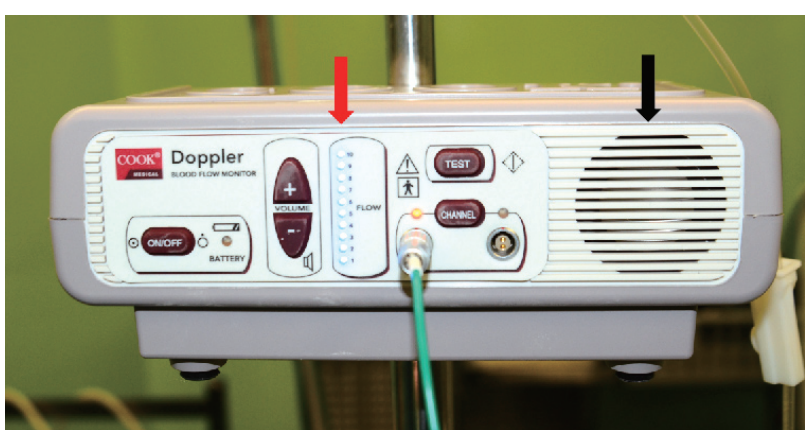

Fig. 4. The implantable Doppler system readily indicates changes in free flap perfusion and makes free flap monitoring easy by providing visible (red arrow) and audible (black arrow) real-time signal information. 


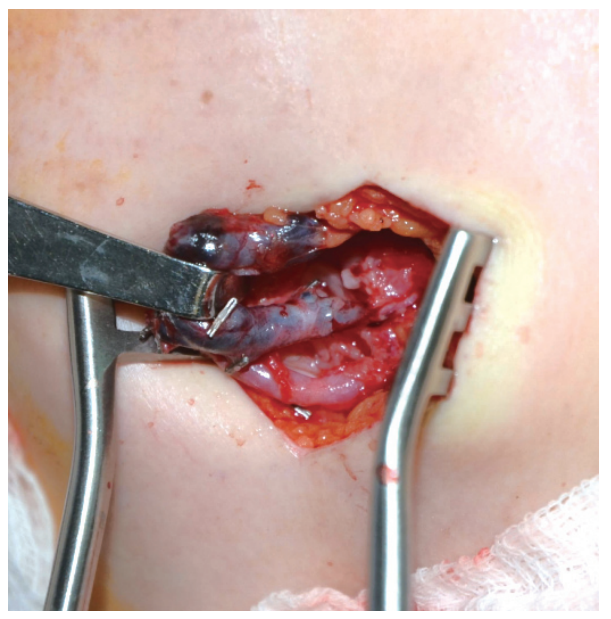

Fig. 5. The patient's caregiver notified of sudden change in Doppler signal. Upon expidited exploration, the vein was found to have a venous thrombus from 1 to $3 \mathrm{~cm}$ distal to the anastomotic point.

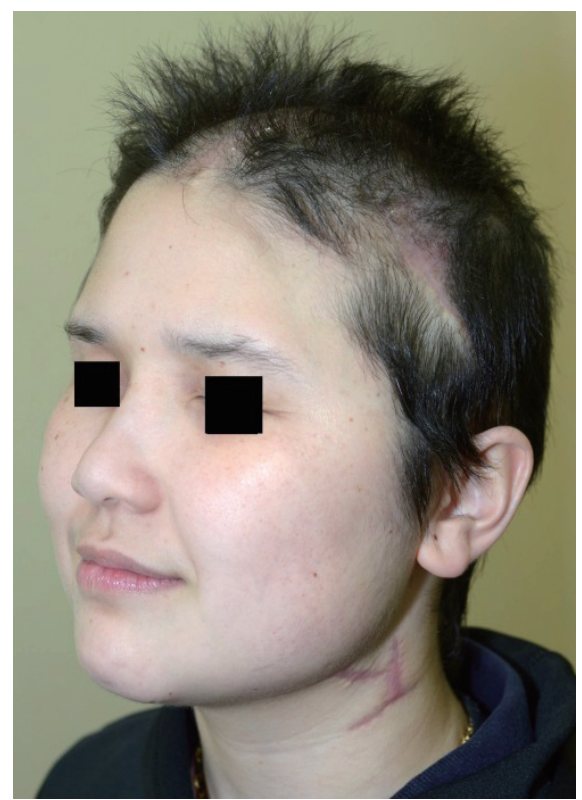

Fig. 6. Postoperative view at 3 months.

\section{DISCUSSION}

The implantable Doppler system designed by Swartz et al. provides a reliable way of monitoring buried flaps [3]. Over several decades, the device has gained widespread attention as a free flap monitoring device for use- - both as a standalone method and in combination with other methods. Conventional free flap monitoring techniques require an external component, whereas an im- plantable monitor readily indicates the changes in free flap perfusion, especially in buried flaps used in head and neck reconstruction [4]. The implantable portion of the system-the ultrasound probe- is constructed from a silicon cuff wrapped around the distal portion of venous anastomosis area and stays safely in place without disturbing the blood flow. The signal is delivered through the wire, which is brought through the skin incision and is connected to the portable monitor. The wire is detached from the silastic cuff and withdrawn at around 5 to 7 days, provided that the vascular flow has been reliable through this time [1].

There are several benefits in using the implantable Doppler device. First, the device is minimally invasive and can be secured to the vascular pedicle. The external monitor is portable and convenient for the nursing staff to use without disturbing the patient. In addition, it is theoretically possible for the implantable Doppler device to reveal compromised circulation earlier than physical findings or the decline of a cutaneous arterial signal in an outflow limited situation. Most importantly, the implantable Doppler device can be used to detect vascular events for deep or buried free flaps. Rozen et al. [2] argued that the implantable Doppler had a high positive predictive value and a low false-positive rate. In addition, Paydar et al. [1] reported that the implantable Doppler had a high sensitivity, a high negative predictive value, and a low false-negative rate. However, there are still limitations to the technique due to the controversy regarding whether its use actually increases flap survival rates $[1,4,5]$. An important downside, as noted by others, is the high rate of false-positive readings (up to 88 percent) [6].

In our case, two things needed to be considered before the flap transfer. First, the patient had already undergone two cranioplasty operations, and the superficial temporal vasculature had been tempered with. The recipient vessels in the face required a long vascular pedicle with the problems associated with such flap configuration. Second, the left temporal region remained depressed and infected despite the previous operations. The patient and her caregivers were extremely nervous and irritable. For this reason, we decided to utilize the implantable Doppler device. We thought that it could help not only to detect a potential thrombosis or insufficient blood flow early, but also to reassure the patient and her 
caregivers by explaining its benefits.

The implantable Doppler device allows for real-time detection of vascular occlusion during the vital early postoperative period. Loss or change in the Doppler signal can signify a loss of flow through the venous or arterial pedicle, prompting early re-exploration and a potential salvage of a flap. Moreover, patient's family, friends, or patients themselves can participate in monitoring the flap through this minimally invasive, simple device.

\section{REFERENCES}

1. Paydar KZ, Hansen SL, Chang DS, Hoffman WY, Leon P. Implantable venous Doppler monitoring in head and neck free flap reconstruction increases the salvage rate. Plast Reconstr Surg 2010;125:112934.

2. Rozen WM, Chubb D, Whitaker IS, Acosta R. The efficacy of postop- erative monitoring: a single surgeon comparison of clinical monitoring and the implantable Doppler probe in 547 consecutive free flaps. Microsurgery 2010;30:105-10.

3. Guillemaud JP, Seikaly H, Cote D, Allen H, Harris JR. The implantable Cook-Swartz Doppler probe for postoperative monitoring in head and neck free flap reconstruction. Arch Otolaryngol Head Neck Surg 2008;134:729-34.

4. Smit JM, Werker PM, Liss AG, Enajat M, de Bock GH, Audolfsson T, Acosta R. Introduction of the implantable Doppler system did not lead to an increased salvage rate of compromised flaps: a multivariate analysis. Plast Reconstr Surg 2010;125:1710-7.

5. Butler CE, Hanasono MM. Discussion. Introduction of the implantable Doppler system did not lead to an increased salvage rate of compromised flaps: a multivariate analysis. Plast Reconstr Surg 2010;125: 1718-9.

6. Rosenberg JJ, Fornage BD, Chevray PM. Monitoring buried free flaps: limitations of the implantable Doppler and use of color duplex sonography as a confirmatory test. Plast Reconstr Surg 2006;118:109-13. 